\title{
Effects of Cordyceps Militaris Polysaccharide on Egg Production, Egg Quality and Caecal Microbiota of Layer Hens
}

\author{
Xiaochen Chen ${ }^{1}$, Yaowen Zhang ${ }^{1}$, Wenfeng $\mathrm{Ma}^{1}$, Yanzi Zhu ${ }^{1}$, Xin $\mathrm{Wu}^{2}$ and Zhanbin Wang ${ }^{1 *}$ \\ ${ }^{I}$ Henan Provincial Academician Workstation of Feed Resource Development and Healthy Livestock, Department of Animal Science \\ and Technology, Henan University of Science and Technology, Luoyang, China; \\ ${ }^{2}$ Observation and Experiment Station of Animal Nutrition and feed science in South-Central China, Ministry of Agriculture, Hunan \\ Provincial Engineering Research Center for Healthy Livestock and poultry production, Key Laboratory of Agro-Ecological Processes \\ in Subtropical Region, Institute of Subtropical Agriculture, Chinese Academy of Sciences, Changsha, China. \\ *Correspondence author’s Email: wangzhanbin3696@126.com; ORCID: 0000-0001-7466-7754
}

Received: 06 Jan. 2020

Accepted: 11 Feb. 2020

\begin{abstract}
The present study was conducted to determine the effects of the diet supplementation of laying hens with Cordyceps Militaris Polysaccharide (CMP) on egg production and quality, and also caecal microbiota. A total of 360 Hy-Line Brown laying hens with 72-week-old were divided into three groups with four replicates of 30 birds each. The laying hens were fed with basal diet (control group), basal diet $+100 \mathrm{mg} \mathrm{CMP} / \mathrm{kg}$ (group 1) and basal diet $+200 \mathrm{mg} \mathrm{CMP} / \mathrm{kg}$ (group 2). The experiment lasted 45 days. Eggs were collected daily and caecal samples were collected at the end of the experiment. Results showed that dietary supplementation with CMP did not affect albumen height, shape index, Haugh units, eggshell breaking strength and eggshell thickness. The laying rate significantly increased and the feedegg ratio decreased in groups 1 and 2, the average daily egg weight significantly increased in group 2 compared with those in the control group. However, insignificant differences were found in broken egg rate and mortality among the three groups. The differences in caecal microbiota between group one and the control group were significant. The relative abundance of Firmicutes, Bacteroidetes and Proteobacteria at the phylum level, Christensenellaceae and Veillonellaceae at the family level, and the no rank_ Ruminococcaceae, Phascolarctobacterium and no rank Christensenellaceae at the genus level changed significantly in group one compared with those in the control group. In conclusion, dietary supplementation with 100 and $200 \mathrm{mg} \mathrm{CMP} / \mathrm{kg}$ could improve product performance and affect the caecal microbial community structure of laying hens during the late laying period.
\end{abstract}

Key words: Cordyceps militaris polysaccharide, Egg production, Egg quality, Gut microbiome, Hens

\section{INTRODUCTION}

Antibiotics, a feed additive to improve animal production performance and disease resistance, are widely used in animal production. However, abuse of antibiotics during feeding had led to drug resistance of pathogens, which led to an imbalance in the intestinal flora of livestock and poultry (Chang et al., 2015). Antibiotics residues in livestock products and caused environmental pollution (Martinez, 2009). The researchers were therefore trying to find better antibiotic alternatives for laying hens.

Cordyceps Militaris Polysaccharide (CMP), one of the most important active substances from C. militaris, was composed of mannose, cordycepin, adenosine, arabinose, and fucose. CMPs extracted from C. militaris and C. sinensis had the same effect, and C. sinensis was rare, so Cordyceps polysaccharide was often extracted from C. militaris (Das et al., 2010). Studies had shown that
Cordyceps polysaccharides had some positive effects on improving immunity, reducing blood lipids and protecting the liver and the kidney (Cheung, et al., 2009; Lee, et al., 2010a; Shin, et al., 2010). Cordyceps polysaccharides, as a feed additive had received wide attention in Asia due to their diverse functions in the livestock industry. For example, Cordyceps polysaccharides could promote the early growth of chickens, increase the feed conversion ratio and enhance the daily growth gain of broilers (Han et al., 2015). C. militaris waster medium increased the egg production rate and decreased the feed- egg ratio $(\mathrm{F} / \mathrm{G})$ (Wang et al., 2015). Hot-water extract from the mycelia of C. sinensis maintained the better microbial flora from the intestines of chickens and improved the growth performance and health index of chickens (Koh et al., 2003).

Microbial communities in the intestine played a 
crucial role in the health and function of hosts. Animal intestinal flora was a complex and diverse system and could easily be influenced by many factors, such as environment, age, nutrition, feed additive and hygiene level. Nutritional differences were the main cause of the overall variations, suggesting that food components could influence the composition and diversity of the intestinal flora (Zhang et al., 2010). Chicken egg production and quality generally decreased with age, reducing the agricultural profits of poultry farmers. It was therefore important to keep the egg production at a high level. There had been few reports on the effects of CMP as a feed additive on poultry nutrition, particularly on the intestinal flora in the late laying period. Hence, this study was conducted to determine the effects of CMP supplementation on egg production and quality, and also caecal microbiota during the late laying period of chickens and to provide valuable information on the use of CMP as feed additives.

\section{MATERIALS AND METHODS}

\section{Ethical approval}

This research did not involve the introduction of any intervention on hens. The data collcetion was obtained with humanly handled, which according of animal care and welfare standard of The People's Republic of China. Animal experimentation procedures were approved by the Institutional Animal Care and Use Committee of Henan University of Science and Technology, Luoyang, China.

\section{Preparation of Cordyceps militaris polysaccharide}

Freeze-dried C. militaris polysaccharide was purchased from Yangshao Biology and Chemical Engineering Company, Mianchi City, Henan Province, China.

\section{Laying hens, diets and experimental design}

A total of 360 Hy-Line Brown laying hens at 72week of age were assigned to three groups with four replicates of 30 hens in each group. The hens were housed in 120 cages sized $64 \mathrm{~cm} \times 35 \mathrm{~cm} \times 35 \mathrm{~cm}$ (three hens each) During the experiment, the hens were free to obtain feed and water and were exposed to a light dark cycle of 16 hours: 8 hours. The average room temperature was $20 \pm$ $3^{\circ} \mathrm{C}$. Before the experiment started, there was no differences in the egg production rate between the three groups. The laying hens were fed basal diet (control group), and basal diet $+100 \mathrm{mg} / \mathrm{kg} \mathrm{CMP} \mathrm{(group} \mathrm{1)} \mathrm{and}$ basal diet $+200 \mathrm{mg} / \mathrm{kg}$ CMP (group 2). The entire process included a 7-day pre-experiment and a 45-day formal experiment. The diets consisted of corn-based basal rations formulated according to the Management Guide of National Research Council (NRC, 1994) to meet the nutritional requirements of laying hens. Composition of basal feed and nutrients content in proximate analysis were showed in Table 1.

Table 1. Composition and nutrients content of basal feed

\begin{tabular}{lc}
\hline Feedstuffs ingredients & Percentage (\%) \\
\hline Corn grain & 64.50 \\
Soybean meal & 13.00 \\
Rapeseed meal & 4.00 \\
Limestone & 8.00 \\
Premix ${ }^{1}$ & 5.00 \\
Wheat bran & 5.00 \\
Soybean oil & 0.50 \\
Total & 100 \\
\hline Analyzed nutrient composition & Content \\
Dry matter (\%) & 88.89 \\
Ash (\%) & 13.87 \\
Crude Fiber (\%) & 4.17 \\
Crude Protein (\%) & 19.90 \\
Crude Fat (\%) & 4.59 \\
Gross Energy (MJ/ kg) & 16.58 \\
Metabolizable Energy (MJ/ kg) & 11.61 \\
\hline ' = Provide per kg of diet: vitamin A, 4000 IU; vitamin D3, 1200 IU; \\
vitamin E, 6 mg; vitamin B1, 1.4 mg; vitamin B2, 3 mg; vitamin B6, 1.0 \\
mg; vitamin B12, 0.01 mg; pantothenic acid, 7.5 mg; choline chloride, \\
500 mg; biotin, 0.15 mg; Ca,7500 mg; P, 3000 mg; Mn, 72 mg; Zn, 56 \\
mg; Fe, 60 mg; Cu, 25 mg; I, 0.50 mg; Se, 0.10 mg. ${ }^{2}=$ Metabolizable \\
energy was calculated from data provided by Feed Database in China \\
2009. MJ/ kg = Mega Joule per kilogram \\
\end{tabular}

\section{Egg production}

During the experimental period, daily records of egg production, egg cracking, egg weight and mortality of hens and records of feed consumption were maintained. The Lay rate (including cracked eggs), average daily egg weight, cracked egg rate, and feed to egg ratio were analyzed.

\section{Egg quality}

Six saleable eggs (no shell defects or cracks) were randomly selected from each replication on day 45 and used to determine egg quality, egg albumen height and Haugh units were determined with a digital egg tester (TSS, England, UK), eggshell breaking strength was identified with a strength instruction (In-spec 2200, Instron Corporation, Canton, Massachusetts, USA), egg shape index was assessed using the following formula including vertical diameter/transect diameter, egg diameter 
was assessed with a vernier caliper and eggshell thickness was assessed with a Spiral range finder.

\section{Caecal microbiome}

Four hens were randomly selected from the control group and group 2 (one for each replication) and sacrificed on the 45th day. The caecum samples were collected aseptically from each bird, immediately stored at $-80{ }^{\circ} \mathrm{C}$ and processed promptly.

\section{DNA extraction and PCR amplification}

According to the manufacturer's protocol, an E.Z.N.A.® soil DNA kit (Omega Bio-tek, Norcross, GA, USA) was used to extract microbial DNA from cecum samples. Final DNA concentration and purification were determined using a Nano Drop 2000 UV-vis spectrophotometer (Thermo Scientific, Wilmington, USA), and DNA quality was checked through $1 \%$ agarose gel electrophoresis. The hypervariable regions (variable 3variable 4) of the bacterial 16S rRNA gene were amplified with the primers $341 \mathrm{~F}$ (5'-CCTAYGGGRBGCASCAG-3') and 806R (5'-GGACTACNNGGGTATCTAAT-3') by using a thermocycler PCR system (GeneAmp 9700, ABI, USA). The PCR was conducted by denaturising at 95 Centigrade $\left({ }^{\circ} \mathrm{C}\right.$ ) for 5 minutes (min.), followed by 27 cycles at $95{ }^{\circ} \mathrm{C}$ for 30 second (s), $55^{\circ} \mathrm{C}$ for $30 \mathrm{~s}, 72{ }^{\circ} \mathrm{C}$ for $45 \mathrm{~s}$, and a final extension at $72{ }^{\circ} \mathrm{C}$ for $10 \mathrm{~min}$. PCR was performed in triplicate with a $20 \mu \mathrm{L}$ mixture containing 4 $\mu \mathrm{L}$ of $5 \times$ FastPfu Buffer, $2 \mu \mathrm{L}$ of $2.5 \mathrm{mM}$ dNTPs, $0.8 \mu \mathrm{L}$ of each primer $(5 \mu \mathrm{M}), 0.4 \mu \mathrm{L}$ of FastPfu polymerase and $10 \mathrm{ng}$ of template DNA. PCR products were electrophoresed using a $2 \%$ agarose gel, purified using AxyPrep DNA gel extraction kit (Axygen Biosciences, Union City, CA, USA), and quantified using QuantiFluor ${ }^{\mathrm{TM}}$-ST (Promega, USA) in accordance with the manufacturer's protocol.

\section{Illumina MiSeq sequencing}

Purified amplificates were separated pooled in equimolar and paired-end sequenced $(2 \times 300)$ on an Illumina MiSeq platform (Illumina, San Diego, USA) according to the standard protocols of Majorbio BioPharm Technology Co., Ltd. (Shanghai, China). The raw data was stored into the NCB1Sequence Read Archive database (accession nos. SRP154946 and PRJNA555247).

\section{Sequencing data processing}

Trimmomatic were used to demultiplex and do quality-filter for the raw fastq files, then merged them by FLASH according to the following criteria. First, reads were truncated at any site receiving an average quality score of $<20$ over a 50 bp sliding window; then, primers were exactly matched, allowing two nucleotide mismatching, and the reads containing ambiguous bases were removed; eventually, sequences with overlaps longer than 10 bp were merged on the basis of their overlap sequence.

Using UPARSE version 7.1 the operational taxonomic units (OTUs) were clustered with a $97 \%$ similarity cut-off, the UCHIME were used to identify and remove chimeric sequences. RDP Classifier algorithm were used to analyse the taxonomy of each 16S rRNA gene sequence, which against the Silva (SSU123) $16 \mathrm{~S}$ rRNA database with a confidence threshold of $70 \%$.

\section{Data analysis}

Data were analyzed by one-way ANOVA using SPSS 22.0 (SPSS Inc., Chicago, IL, USA). The significance of the mean differences between the groups was identified by a Tukey test. Data significance was determined at $\mathrm{p}<$ 0.05. Alpha diversity and dilution curve analyses consisted of community diversity (simpson and shannon), richness (sobs, chao and ace) were performed using mothur (a 16S rRNA clustering tool) based on a single summary command. Beta diversity analysis was carried out to investigate the diversity between the samples. Principal component analysis (PCA) was conducted with mothur to describe the distances between the samples, and a permutative multivariate analysis of variance (PERMANOVA) was performed to analyze the significance of the OTU levels. Wilcoxon rank sum test was used to determine the significant differences in the relative abundance of bacteria at phylum and genus level between the CMP and the control group.

\section{RESULTS}

\section{Egg production}

Table 2 shows the laying performance of hens. The egg production increased significantly and the feed-egg ratio decreased ( $p<0.01)$ in groups 1 and 2 compared to those in the control group. The average daily egg weight increased significantly in group $2(\mathrm{p}<0.05)$. However, insignificant differences in the cracked egg rate and mortality were found among the three groups ( $p>0.05)$. The egg production in group 2 increased significantly compared to group 1 ( $\mathrm{p}<0.01)$.

\section{Egg quality}


Table 3 shows the egg quality of hens. Insignificant differences were observed in the egg albumen height, the egg shape index, the Haugh units, eggshell breaking strength and eggshell thickness between three groups $(\mathrm{p}>$ $0.05)$.

\section{Caecal microbiota}

A total of 380,227 DNA sequence read with an average length of $415 \mathrm{bp}$ were generated from all of samples. The average number of sequence readings per sample was 47,528 , with a minimum number of 35,454 and a maximum number of 58,207 . The sequences were further clustered into 749 OTUs by using a $97 \%$ similarity cut off. The sparse curve generated by the OTU shows that all samples achieved a high sampling coverage ( 99\%) (Figure 1). Table 4 shows the alpha diversity indices of caecal microbiota. The average values of ace, chao and sobs in the CMP group were significantly lower than those in the control group.

The PCA on the OTU level showed the changes in the community structure (Figure 2). The microbial community of the CMP group was clearly separated from that of the control group. The results revealed a significant difference in the bacterial structure in the caecum between the CMP and control groups (PERMANOVA, $\mathrm{p}=0.026$ ).

Figure 3 and table 5 demonstrate the phylum distributions of the microbial composition. In the CMP group and the control group, the main flora were
Firmicutes, Bacteroidetes and Proteobacteria. Firmicutes significantly decreased in the CMP group compared with that in the control group $(\mathrm{p}<0.05)$. In contrast, the proportions of Bacteroidetes in the CMP group increased compared to those in the control group $(\mathrm{p}<0.05)$. The two phyla with a low relative abundance (Synergistetes and Elusimicrobia) decreased significantly in the CMP group compared to those in the control group $(\mathrm{p}<0.05)$.

The family distribution results of the microbial composition are shown in figure 4 and table 6 . The main families of the CMP and control groups were Ruminococcaceae, Bacteroidales and Lachnospiraceae. Among them, Ruminococcaceae was the most abundant. The two families with a low relative abundance (Veillonellaceae and Christensenellaceae) decreased significantly in the CMP group compared to those in the control group $(\mathrm{p}<0.05)$.

The microbial composition was also comparable at the genus level (Figure 5 and table 7). The main genera of the CMP and control groups were norank Bacteroidales, norank Ruminococcaceae, Bacterorides Bacteroidaceae and Oscillospira. Among these genera, the relative abundance of norank Ruminococcaceae decreased significantly in the CMP group compared to those in the control group $(p<0.05)$. The two genera with a low relative abundance (Phascolarctobacterium and norank Christensenellaceae) decreased significantly in the CMP group compared to those in the control group ( $\mathrm{p}<0.05$ ).

Table 2. Effects of Cordyceps militaris polysaccharide on laying performance in 72-week-old laying hen at Louyang,China.

\begin{tabular}{|c|c|c|c|c|c|c|}
\hline Items ${ }^{1}$ & Treatment Groups & Control & Group 1 & Group 2 & SEM $^{2}$ & $P$ value \\
\hline Egg production (\%) & & $79.95^{\mathrm{c}}$ & $83.26^{\mathrm{b}}$ & $85.55^{\mathrm{a}}$ & 0.508 & $<0.01$ \\
\hline Cracked egg rate $(\%)$ & & 0.26 & 0.39 & 0.22 & 0.084 & 0.45 \\
\hline Average daily egg weight (g) & & $64.14^{\mathrm{b}}$ & $64.97^{\mathrm{ab}}$ & $65.62^{\mathrm{a}}$ & 0.221 & 0.02 \\
\hline Feed to egg ratio & & $2.17^{\mathrm{a}}$ & $1.96^{\mathrm{b}}$ & $1.98^{\mathrm{b}}$ & 0.023 & $<0.01$ \\
\hline Mortality (\%) & & 4.17 & 3.33 & 4.17 & 0.804 & 0.71 \\
\hline
\end{tabular}

${ }^{\mathrm{T}}$ Control, Group 1, Group 2 with 0, 100, $200 \mathrm{mg} \mathrm{CMP} / \mathrm{kg}$, respectively; ${ }^{2} \mathrm{SEM}=$ pooled standard error of mean. ${ }^{\mathrm{a}, \mathrm{b}}=$ Means not sharing the same superscripts in a row differ significantly $(\mathrm{p}<0.05)$.

Table 3. Effects of Cordyceps Polysaccharide on egg quality in 72-week-old laying hen at Louyang, China.

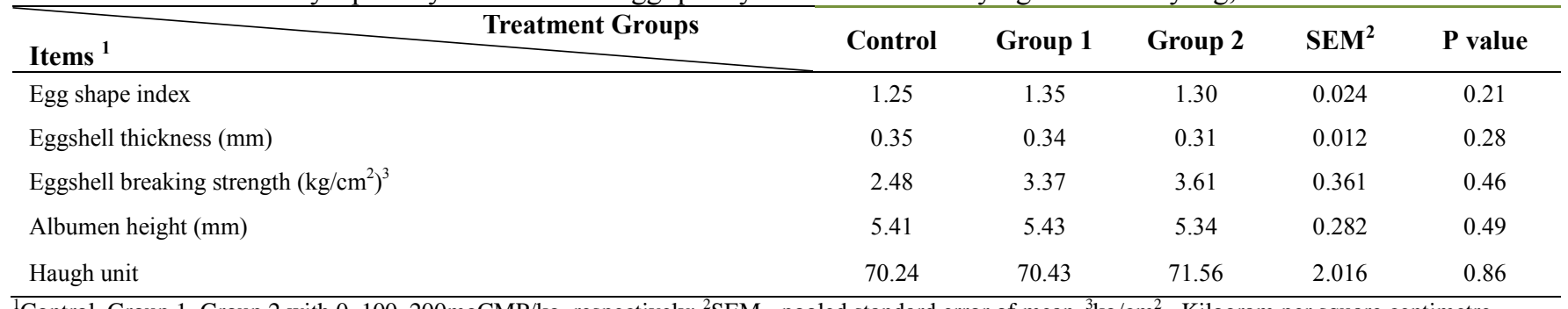

${ }^{1}$ Control, Group 1, Group 2 with 0, 100, 200mgCMP/kg, respectively; ${ }^{2} \mathrm{SEM}=$ pooled standard error of mean. ${ }^{3} \mathrm{~kg} / \mathrm{cm}^{2}=$ Kilogram per square centimetre 
Table 4. Alpha Indices data of caecum samples in 72-week-old laying hen at Louyang,China.

\begin{tabular}{|c|c|c|c|c|c|}
\hline Estimators & CON-Mean ${ }^{1}$ & CON-Sd ${ }^{2}$ & CMP-Mean & CMP-Sd & P Value \\
\hline Ace & 710.73 & 15.594 & 629.72 & 42.39 & 0.012 \\
\hline Chao & 712.82 & 12.958 & 638.82 & 45.648 & 0.021 \\
\hline Coverage & 0.99834 & 0.0007961 & 0.9979 & 0.00093558 & 0.502 \\
\hline Shannon & 5.1584 & 0.080538 & 4.9022 & 0.25779 & 0.107 \\
\hline Simpson & 0.011827 & 0.0018573 & 0.017143 & 0.0077245 & 0.230 \\
\hline Sobs & 664.75 & 27.244 & 577.5 & 49.89 & 0.022 \\
\hline
\end{tabular}

${ }^{\mathrm{I}}$ Mean $=$ the mean number; ${ }^{2} \mathrm{Sd}=$ the standard deviation; $\mathrm{CON}=$ control group $\mathrm{CMP}=$ Cordyceps Militaris Polysaccharide

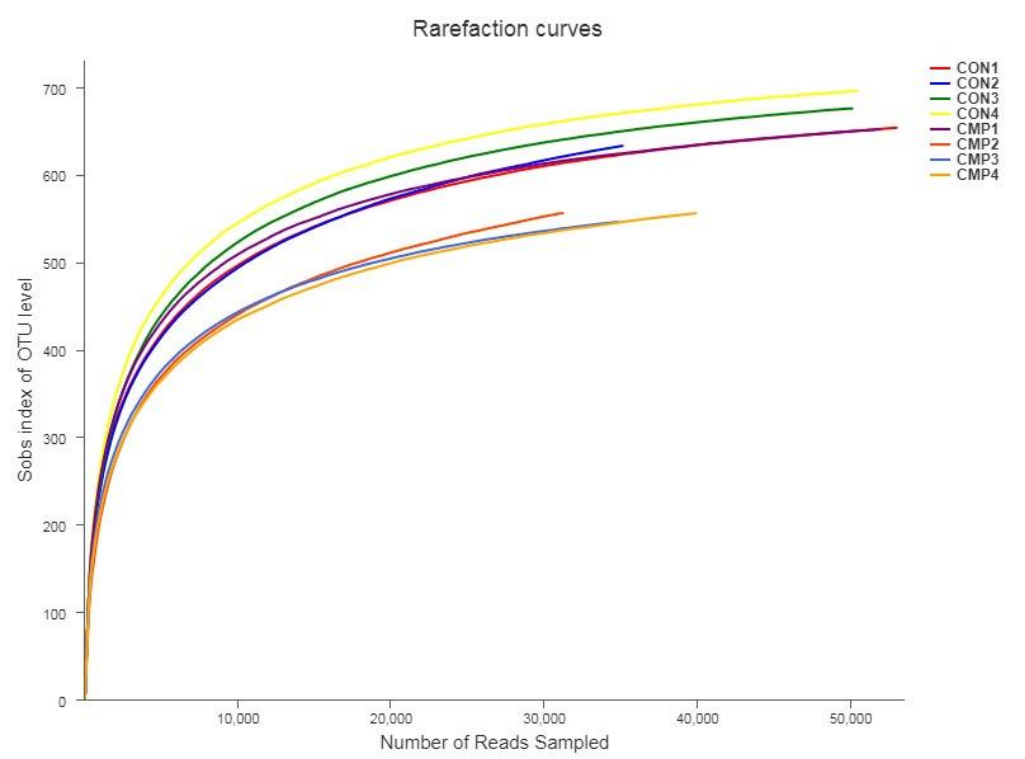

Figure 1. Rarefaction curves of samples $(n=8)$ clustered at $97 \%$ sequences identity. OTU= Operational Taxonomic Unit; $\mathrm{CON}=$ control group; $\mathrm{CMP}=$ Cordyceps Militaris Polysaccharide.

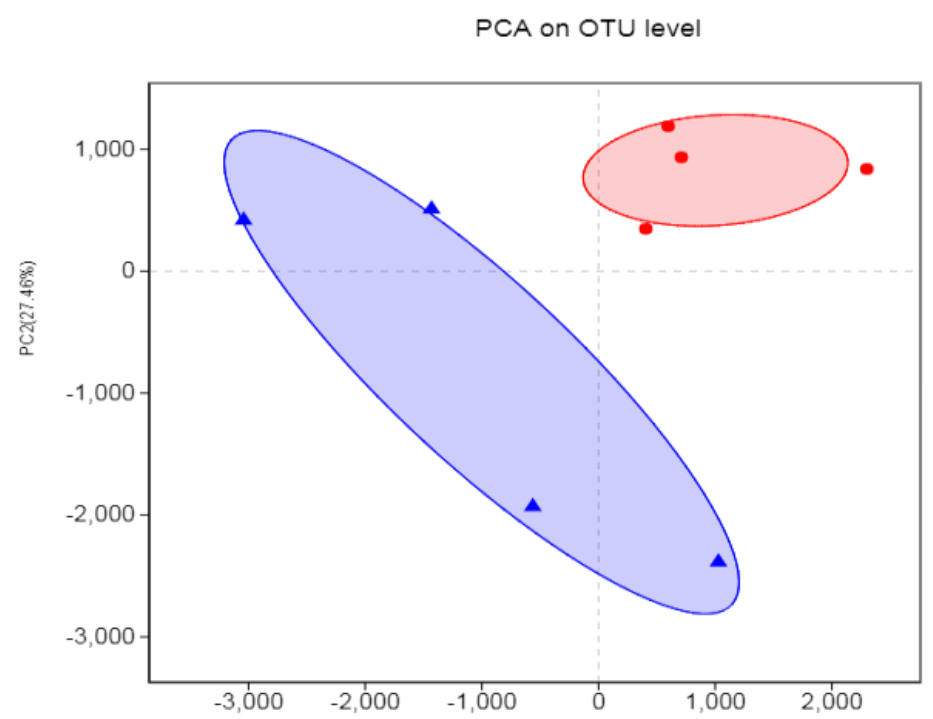

Figure 2. Principal Component Analysis (PCA) of the community membership using Bray-Curtis distance. CON = control group; $\mathrm{CMP}=$ Cordyceps Militaris Polysaccharide. The abscissa and ordinate represent the two selected principal components, and the percentage represents the contribution of the principal component to the difference in sample composition. Points of 
different colors and shapes represent samples of different groups, and the closer the two sample points are, the more similar the composition of the two samples species is.

\section{Wilcoxon rank-sum test bar plot on Phylum level}

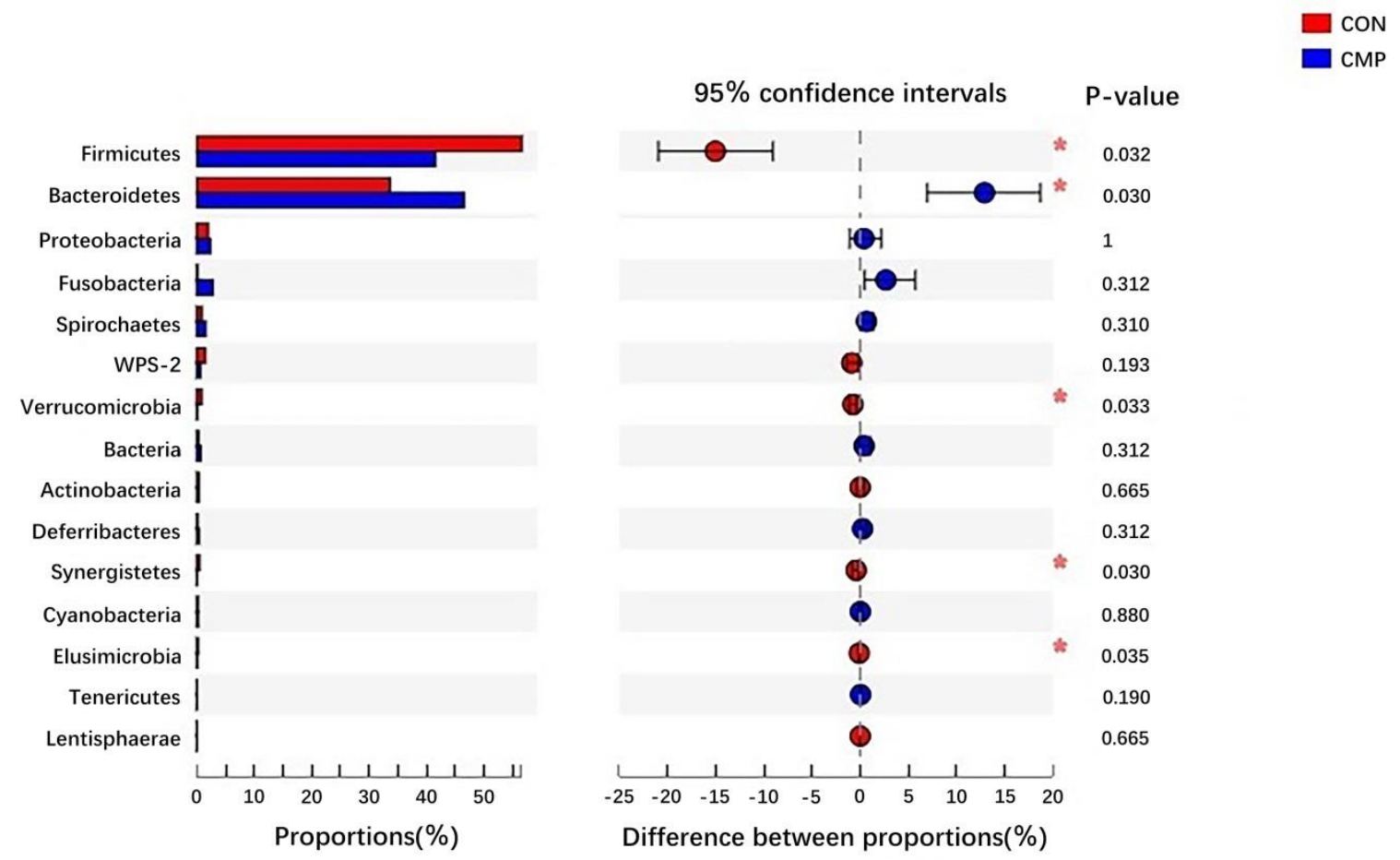

Figure 3. Welch's t-test bar plot on Phylum level. $\mathrm{CON}=$ control group; $\mathrm{CMP}=$ Cordyceps Militaris Polysaccharide. The ordinate (left) represents the phyla name and the ordinate (right) represents the $P$-Value.

\section{Wilcoxon rank-sum test bar plot on Family level}

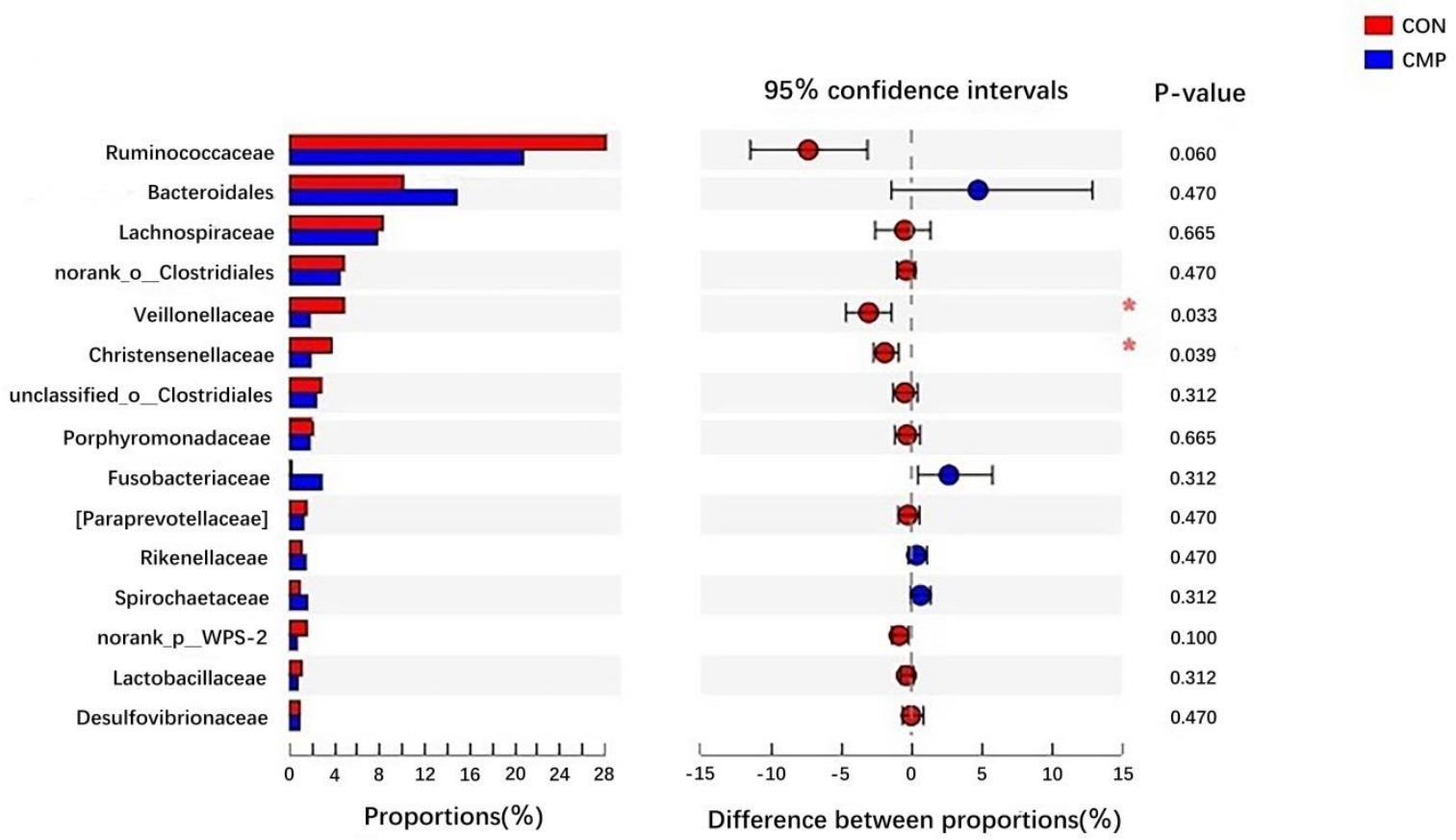


Figure 4. Welch's t-test bar plot on Family level. $\mathrm{CON}=$ control group; $\mathrm{CMP}=$ Cordyceps Militaris Polysaccharide. The ordinate (left) represents the family name and the ordinate (right) represents the $p$-Value.

Wilcoxon rank-sum test bar plot on Genus level

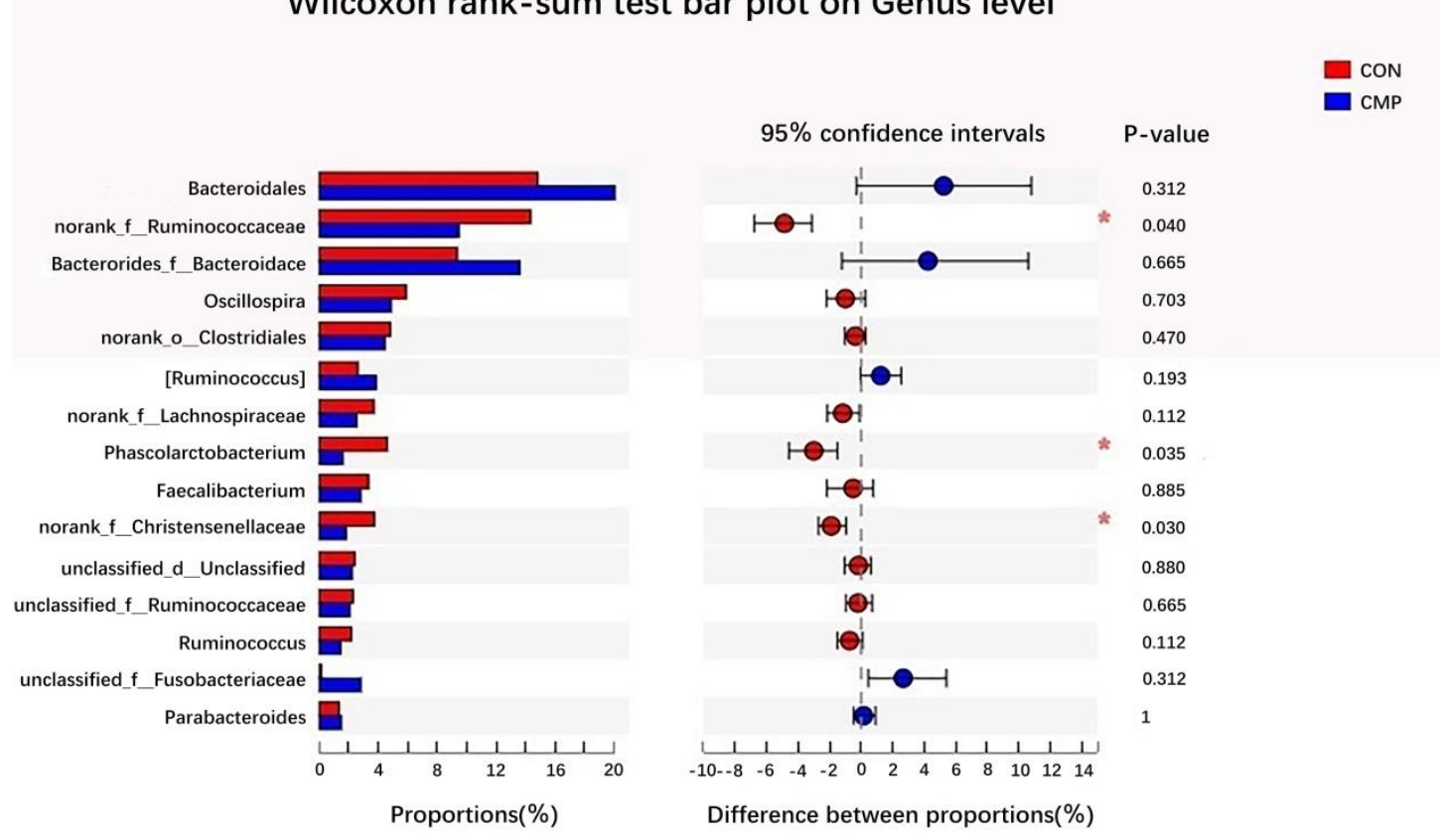

Figure 5. Welch's t-test bar plot on Genus level. $\mathrm{CON}=$ control group; CMP = Cordyceps Militaris Polysaccharide. The ordinate (left) represents the genus name and the ordinate (right) represents the p-Value.

Table 5. Wilcoxon rank sum test data on Phylum level (cut off at 1\%).

\begin{tabular}{|c|c|c|c|c|c|}
\hline Species name & CMP-Mean $^{1}(\%)$ & $\operatorname{CMP}_{-S^{2}}^{2}(\%)$ & CON-Mean (\%) & CON-Sd (\%) & $P$ value \\
\hline Bacteroidetes & 46.57 & 6.96 & 33.66 & 1.768 & 0.030 \\
\hline Fusobacteria & 2.827 & 3.139 & 0.1601 & 0.06761 & 0.312 \\
\hline Spirochaetes & 1.559 & 0.7397 & 0.9031 & 0.3315 & 0.312 \\
\hline
\end{tabular}

Table 6. Wilcoxon rank sum test data on Family level (cutoff at 1\%).

\begin{tabular}{|c|c|c|c|c|c|}
\hline Species name & CMP-Mean ${ }^{1}(\%)$ & $\operatorname{CMP}_{-S^{2}}{ }^{2}(\%)$ & CON-Mean (\%) & CON-Sd (\%) & $P$ value \\
\hline Bacteroidales & 14.81 & 8.024 & 10.07 & 0.7542 & 0.470 \\
\hline norank_o__Clostridiales & 4.45 & 0.5395 & 4.818 & 0.5201 & 0.470 \\
\hline Veillonellaceae & 1.784 & 0.9881 & 4.831 & 1.653 & 0.030 \\
\hline Porphyromonadaceae & 1.769 & 1.058 & 2.084 & 0.2 & 0.665 \\
\hline Fusobacteriaceae & 2.827 & 3.139 & 0.1601 & 0.0676 & 0.312 \\
\hline Paraprevotellaceae & 1.233 & 0.8325 & 1.478 & 0.4542 & 0.470 \\
\hline Rikenellaceae & 1.433 & 0.7874 & 1.072 & 0.1486 & 0.470 \\
\hline Spirochaetaceae & 1.559 & 0.7397 & 0.9031 & 0.3315 & 0.312 \\
\hline
\end{tabular}




\begin{tabular}{|c|c|c|c|c|c|}
\hline Lactobacillaceae & 0.7092 & 0.5013 & 1.071 & 0.2273 & 0.312 \\
\hline \multicolumn{6}{|c|}{$\begin{array}{l}{ }^{1} \text { Mean }=\text { the percentage of the mean number of Family; }{ }^{2} \mathrm{Sd}=\text { the percentage of star } \\
\text { Cordyceps Militaris Polysaccharide. } \\
\text { Table7. Wilcoxon rank sum test data on Genus level (cutoff at } 1 \% \text { ). }\end{array}$} \\
\hline norank_o__Bacteroidales & 20.05 & 6.837 & 14.82 & 0.3941 & 0.312 \\
\hline norank_f_Ruminococcaceae & 9.476 & 1.981 & 14.35 & 0.838 & 0.040 \\
\hline norank_o_Clostridiales & 4.45 & 0.5395 & 4.818 & 0.5201 & 0.470 \\
\hline Ruminococcus & 3.85 & 1.248 & 2.612 & 0.8816 & 0.193 \\
\hline norank_f_Lachnospiraceae & 2.534 & 0.9579 & 3.701 & 0.8048 & 0.112 \\
\hline Phascolarctobacterium & 1.611 & 0.8582 & 4.618 & 1.682 & 0.035 \\
\hline Faecalibacterium & 2.824 & 1.488 & 3.339 & 0.7728 & 0.885 \\
\hline norank_f_Christensenellaceae & 1.832 & 0.9219 & 3.735 & 0.4204 & 0.030 \\
\hline Parabacteroides & 1.501 & 0.8043 & 1.349 & 0.1146 & 1.000 \\
\hline norank_f_Rikenellaceae & 1.432 & 0.7874 & 1.071 & 0.1485 & 0.470 \\
\hline Treponema & 1.559 & 0.7397 & 0.9031 & 0.3315 & 0.312 \\
\hline norank_f_Paraprevotellaceae & 1.122 & 0.7804 & 1.197 & 0.3533 & 0.470 \\
\hline unclassified_f_LLachnospiraceae & 0.8786 & 0.1701 & 1.352 & 0.2803 & 0.061 \\
\hline $\begin{array}{l}\text { norank_p_WPS-2 } \\
\text { unclassified f B Bacteroidaceae }\end{array}$ & 0.6253 & 0.6725 & 1.501 & 0.2236 & 0.194 \\
\hline _o__Bacteroidales & 1.219 & 0.7175 & 0.7158 & 0.132 & 0.312 \\
\hline Lactobacillus & 0.7086 & 0.5018 & 1.07 & 0.2276 & 0.312 \\
\hline
\end{tabular}

\section{DISCUSSION}

\section{Effects of CMP on egg production and egg quality}

It has been clinically confirmed that $C$. militaries had several functions, such as antitumor, anti-inflammatory and antioxidant effects (Chen, et al., 2010; Lee, et al., 2010b; Li, et al., 2015). Cordyceps polysaccharide isolated from $C$. militaries could suppress a cyclophosphamideinduced immune response to improve lymphocyte and macrophage activities, increased superoxide dismutase, catalase and glutathione peroxidase levels and reduced malondialdehyde content in the blood in mice (Wang, et al., 2012). However, information on the impact of CMP supplementation on laying hens is limited. Researchers emphasized that feeding laying hens with $C$. militaries waster medium decreased significantly feed conversion ratio (FCR) and increased egg production. They also showed that ascorbic acid and $C$. militaries waster mediuman have approximately the same reducing power (Wang et al., 2015 ) Cifticiet al. indicated that ascorbic acid can partially inhibit adverse oxidative protein denaturation and improve nutrient digestibility and FCR (Ciftici,et al.,2005). As such, the feed-egg ratio of the hens receiving the added CMP in the present study was likely to decrease and egg production to increased.

Eggshell thickness, eggshell strength, Haugh unit and egg shape index were important indicators for egg quality measurement, and these parameters were related to the shelling rate and freshness of eggs. In the present study, the differences in the egg quality between the three groups were insignificant. Similarly, scholars observed that supplementing a diet with $C$. militaries waster medium did not improve eggshell thickness and egg shell strength (Wang et al., 2015).

\section{Effects of CMP on ceacal microbiota}

Chicken intestinal microflora had a positive effect on food intake and the immune system (Shang et al., 2018b). Studies had shown that a well-functioning caecum could cover $10 \%$ of the body's energy needs (Hegde, et al., 1982; Józefiak, et al., 2004).

Our study determined the alpha diversity of microbial communities by measuring Sobs, Shannon's, Simpson's, Ace and Chao indices. Shannon's and Simpson's indices represent the degree of differences in the frequencies of different taxa (diversity), while Sobs, Chao and Ace indices reflect the number of different taxa present in the 
sample (richness). The present study showed the significant differences in the caecal microbial density between the CMP and control groups. Consistent with previous findings (Bederska-ojewska et al., 2017), our results revealed significantly lower averages for microbial diversity and richness in the CMP group compared to those in the control group. Scientists found that the addition of cordycepin had an antimicrobial function similar to tetracycline and chloramphenicol (Ahn et al., 2000). These results indicated that CMP supplementation appeared to reduce the bacterial species in the caecal intestine of chicken. Further studies were needed to investigate the specific mode of action of CMP.

At the phylum level, differences in microbial structures could mostly be attributed to changes in the microbiome in Firmicutes, Bacteroidetes, Synergistetes and Elusimicrobia in the CMP group. In accordance with a previous research (Pan and Yu, 2014), the present study also found that Firmicutes, Bacteroidetes and Proteobacteria accounted for more than $90 \%$ in the chicken caecal microflora. The relative abundance of Bacteroidetes in the CMP group was higher than in the control group, while the Firmicutes content decreased significantly. Bacteroides could digest complex carbohydrates and maintain an intestinal micro-ecological balance (Józefiak et al., 2004; Sears 2005). Bacteroides could promote the development of the immune system (Spence et al., 2006). Bacteroidetes was related to the development of interleukin-17-producing T-helper cells (Mazmanian et al., 2005). Some studies have shown that with the increase of body mass index (BMI), the content of a increases gradually, while the content of B decreases (Koliada et al., 2017). Some studies had found differences between infants, adults and the elderly in relation to the Firmicutes to Bacteroidetes ratio. Among them, adults had the highest value (Mariat et al., 2009). Therefore, our results might had a positive effect on the health and fat accumulation of hens during the late laying period. In the present study, we also observed significant differences in the relative abundance of Synergist and Elusimicrobia between the CMP and control groups, but the functions of these phyla were unclear and should be investigated further.

At the family level, the relative abundance of Veillonellaceae and Christensenellaceae decreased significantly in the CMP group, but was low in our experimental hens. Christensenellaceae belongs to the order of the Clostridiales within the Clostridia class of Firmicutes (Morotomi et al., 2012) and had emerged as the intestinal flora component, which the abundance was most influenced by host genetics (Goodrich et al., 2014). Christensenellaceae might be a symbol of the ecosystem of long-lived people (Biagi et al., 2016), Goodrich et al reported the relationship between Christensenellaceae and the weight of humans and mice, that reduced the degree of receptor weight gain (Goodrich et al., 2014). Similarly, in the addition of a Christensella strain in a mouse model, reduced the degree of receptor weight gain (Fischbach and Segre, 2016). The colonisation of Campylobacter jejuni, a foodborne zoonotic pathogen, could increase the relative abundance of Christensenellaceae in a chicken intestine (Alexandre et al., 2015). However, the role of this new family in chicken intestine health was unknown. Veillonellaceae had an excellent ability to ferment lactic acid; it was known as a short-chain fatty acid acetate and propionate producer (Lecomte et al., 2015). Other studies had shown that the number of families defined as the chicken's core microbiome might increase or decrease, but families such as Veilonellaceae had been reported common members of the chicken (Qu et al., 2008; Callaway et al., 2009; Filip, et al., 2011). Veillonelaceae had a similar effect on Campylobacteraceae, so Veillonelaceae could reduce the colonisation of Campylobacteraceae in the chicken intestines (Videnska et al., 2014). However, further studies were needed to investigate the function of Veillonellaceae in the chicken intestine.

At the genus level, the main genera of the CMP and the control groups were norank Bacteroidales, norank Ruminococcaceae, Bacterorides_ Bacteroidaceae and Oscillospira. Scientists reported that the caecum of chickens consisted mainly of Costridia, followed by Lactobacillus and Ruminococcus (Gong et al., 2010). This change might be due to the addition of CMP in the diet. Other factors could also cause these changes. These factors include different types of chicken, age and environmental factors. For example, the abundance of Clostridium increased and the proportion of lactobacill1decreased as the chicken aged (Shang et al., 2018a). Cage type could affect the composition of caecal microflora in laying hens (Filip et al., 2011). Genotype and gender might also affect changes in the intestinal microflora of chicken (Zhao et al., 2013). Norank Ruminococcaceae and norank_Christensenellaceae were unknown bacterial residents, and their relative abundance decreased significantly in the CMP group. Scienticts reported that the caecum was rich in unknown and unclassified bacterial residents (Stanley et al., 2013). The relative abundance of Phascolarctobacterium also decreased significantly in the CMP group compared to 
those in the control group. The Phascolarctobacterium within the Sporomusa branch of the Clostridium/Bacillus subphylum of Gram-positive bacteria and is a neighbor of Acidaminococcus fermentans (Del Dot et al., 1993). Phascolarctobacterium complements the lack of carbohydrate metabolism by increasing lipid metabolism with methylmalonyl-CoA mutase, and methylmalonylCoA carboxyltransferase was the most abundant among proteins involved in lipid metabolism (Polansky et al., 2015). However, evaluating the possible effect of the changes in these genera on the chicken's caecal microbial community was difficult because of the lack of reports about Phascolarctobacterium in the health of chicken intestine.

\section{CONCLUSION}

Dietary supplementation with 100 and $200 \mathrm{mg}$ CMP/kg can improve the laying rat and decrease feed to egg ratio significantly compared to those none-supplemented hens, respectively. Dietary supplementation with CMP could alter significantly the caecal microbiota composition with the changes in the phylum, family and genus levels. These findings provided insights into egg production of chickens and intestinal microbiota modulations in response to different CMP dosages and emphasise the requirement for further research to determine the effects of CMP on the chicken intestine microbiota and predict the response of the microbiota to these agents.

\section{DECLARATIONS}

\section{Acknowledgments}

This study was supported by the National Key Research and Development Program of China (No. 2018YFD500600), the Integration of Industry, Education and Research of Henan Province of China (No. 182107000011) and the Horizontal Tasks of Henan University of Science and Technology (No. 22010055).

\section{Consent to publish}

Not applicable

\section{Competing interests} interests.

The authors declare that they have no competing

\section{Author's contribution}

Xiaochen Chen carried out the molecular genetic studies, participated in the sequence alignment and drafted the manuscript. Yaowen Zhang, and Wenfeng Ma carried out the immunoassays. Xi Wu participated in the sequence alignment. Yanzi Zhu participated in the design of the study and performed the statistical analysis. Zhanbin Wang conceived of the study, and participated in its design and coordination and helped to draft the manuscript. All authors read and approved the final manuscript.

\section{REFERENCES}

Ahn YJ, Park SJ, Lee SG, Shin SC and Choi DH (2000). Cordycepin: Selective growth inhibitor derived from liquid culture of cordyceps $\mathrm{m}$ ilitaris against clostridium spp. Journal of Agricultural \& Food $\begin{array}{lllll}\text { Chemistry, } & 48 & (7): & 2744-2748 . & \text { DOI: }\end{array}$ https://doi.org/10.1021/jf990862n

Alexandre T, Philippe F, étienne Y, Julie A, Ludovic L, Ann L and Markus MH (2015). Chicken caecal microbiome modifications induced by campylobacter jejuni colonization and by a nonantibiotic feed additive. plos one, 10 (7): e0131978. DOI: https://doi:10.1371/journal.pone.013197

Bederska-ojewska D, witkiewicz S and Muszyńska B (2017). The use of basidiomycota mushrooms in poultry nutrition - a review. Animal Feed Science stechnology, 230: 59-69. DOI: https://doi.org/10.1016/j.anifeedsci.2017.06.001

Biagi E, Franceschi C, Rampelli S, Severgnini M, Ostan R, Turroni S, Consolandi C, Quercia S, Scurti M and Monti D (2016). Gut microbiota and extreme longevity. Current Biology, 26 (11): 14801485. DOI: https://doi.org/10.1016/j.cub.2016.04.016

Callaway TR, Dowd SE, Wolcott RD, Sun Y, McReynolds JL, Edrington TS , Byrd JA, Anderson RC, Krueger N and Nisbet DJ (2009). Evaluation of the bacterial diversity in cecal contents of laying hens fed various molting diets by using bacterial tag-encoded flx amplicon pyrosequencing. Poultry science, 88 (2): 298-302. DOI: https://doi.org/10.3382/ps.2008-00222

Chang Q, Wang W, Regev-Yochay G, Lipsitch M and Hanage WP (2015). Antibiotics in agriculture and the risk to human health: How worried should we be? Evolutionary Applications, 8 (3): 240-247. DOI: https://doi.org/10.1111/eva.12185

Chen XL, Wu GH, Gu QX and Huang ZL (2010). Optimization of extraction process of polysaccharides from fruiting body of cordyceps militaris L. Link by orthogonal test. Medicinal Plant, (7). Available https://www.cabdirect.org/?target=\%2fcabdirect $\% 2$ fabstract $\% 2$ f201 13129764

Cheung JKH, Li J, Cheung AWH, Zhu Y, Zheng KYZ, Bi CW, Duan R, Choi RC, Lau DT and Dong TT (2009). Cordysinocan, a polysaccharide isolated from cultured cordyceps, activates immune responses in cultured T-iymphocytes and macrophages: Signaling cascade and induction of cytokines. Journal of Ethnopharmacology, 124 (1): 61-68. DOI: https://doi.org/ 10.1016/j.jep.2009.04.010

Ciftici M, Ertas ON and Guler T (2005). Effects of vitamin E and vitamin $\mathrm{C}$ dietary supplementation on egg production and egg quality of laying hens exposed to chronic heat stress. Revue De Medecine Veterinaire, 156: 107-111. Available at: https://www.revmedvet.com/2005/RMV156_107_111.pdf

Das SK, Masuda M, Sakurai A and Sakakibara M (2010). Medicinal uses of the mushroom cordyceps militaris: Current state and prospects. $\begin{array}{lllll}\text { Fitoterapia, } & 81 & (8): & 961-968 . & \text { DOI: }\end{array}$ https://doi.org/10.1016/j.fitote.2010.07.010

Del Dot T, Osawa R and Stackebrandt E (1993). Phascolarctobacterium faecium gen. Nov, spec. Nov., a novel taxon of the sporomusa group of bacteria. Systematic \& Applied Microbiology, 16 (3): 380-384. DOI: https://doi.org/10.1016/S0723-2020(11)80269-9

Filip VI, Jantina DV, Lotte B, Lars M, Steen N and Karl P (2011). The influence of the cage system and colonisation of salmonella enteritidis on the microbial gut flora of laying hens studied by t-rflp and 454 pyrosequencing. Bmc Microbiology, 11 (1): 187-187. DOI: https://doi.org/10.1186/1471-2180-11-187 
Fischbach MA and Segre JA (2016). Signaling in host-associated microbial communities. Cell, 164 (6): 1288-1300. DOI: https://doi.org/10.1016/j.cell.2016.02.037

Gong J, Si W, Forster RJ, Huang R, Yu H, Yin Y, Yang C and Han Y (2010). 16s rRNA gene-based analysis of mucosa-associated bacterial community and phylogeny in the chicken gastrointestinal tracts: From crops to ceca. Fems Microbiology Ecology, 59 (1): 147-157. DOI: https://doi.org/10.1111/j.1574-6941.2006.00193.x

Goodrich JK, Waters JL, Poole AC, Sutter JL, Koren O, Blekhman R, Beaumont M,Van T.W, Knight R and Bell JT (2014). Human genetics shape the gut microbiome. Cell, 159 (4): 789-799. DOI: https://doi.org/10.1016/j.cell.2014.09.053

Han JC, Qu HX, Wang JG, Yan YF, Zhang JL, Yang L, Zhang M and Cheng YH (2015). Effects of fermentation products of cordyceps militaris on growth performance and bone mineralization of broiler chicks. Journal of Applied Animal Research, 43 (2): 236-241. DOI: https://doi.org/10.1080/09712119.2014.928630.

Hegde SN, Rolls BA and Coates ME (1982). The effects of the gut microflora and dietary fibre on energy utilization by the chick. British Journal of Nutrition, 48 (1): 73-80. DOI: https://doi.org/10.1079/bjn19820089

Józefiak D, Rutkowski A and Martin SA (2004). Carbohydrate fermentation in the avian ceca: A review. Animal Feed Science \& Technology, $113 \quad$ (1): $1-15$. https://doi.org/10.1079/bjn19820089

Koh JH, Suh HJ and Ahn TS (2003). Hot-water extract from mycelia ofcordyceps sinensisas a substitute for antibiotic growth promoters. Biotechnology Letters, 25 (7): 585-590. DOI: https://doi.org/10.1023/a:1022893000418

Koliada A, Syzenko G, Moseiko V and Budovska L (2017). Association between body mass index and firmicutes/bacteroidetes ratio in an adult ukrainian population. Bmc Microbiology, 17 (1): 120. DOI: https://doi.org/10.1186/s12866-017-1027-1

Lecomte V, Kaakoush NO, Maloney CA, Raipuria M, Huinao KD, Mitchell HM and Morris MJ (2015). Changes in gut microbiota in rats fed a high fat diet correlate with obesity-associated metabolic $\begin{array}{llllll}\text { parameters. PLoS ONE, } 10 & \text { (5): } 0126931 . & \text { DOI: }\end{array}$ https://doi.org/10.1371/journal.pone.0126931

Lee JS, Kwon JS, Won DP, Lee JH, Lee KE, Lee SY and Hong EK (2010a). Study of macrophage activation and structural characteristics of purified polysaccharide from the fruiting body of cordyceps militaris. Journal Microbiol Biotechnol, 20 (7): 10531060. DOI: https://doi.org/10.4014/jmb.0910.10022

Lee JS, Kwon JS, Yun JS, Pahk JW, Shin WC, Young LS and Hong EK (2010b). Structural characterization of immunostimulating polysaccharide from cultured mycelia of cordyceps militaris. $\begin{array}{lllll}\text { Carbohydrate } \text { Polymers, } 80 & \text { (4): } 1011-1017 . \quad \text { DOI: }\end{array}$ https://doi.org/10.1016/j. carbpol.2010.01.017

Li Y, Jing Z, Shao PL, Hui F and Quan Z (2015). Quality evaluation of cordyceps through simultaneous determination of eleven nucleosides and bases by rp-hplc. Journal of Separation Science, 29 (7): 953-958. DOI: https://doi.org/10.1002/jssc.200600007

Mariat D, Firmesse O, Levenez F, Guimares V, Sokol H, Doré J, Corthier $\mathrm{G}$ and Furet JP (2009). Thefirmicutes/bacteroidetes ratio of the human microbiota changes with age. Bmc Microbiology, 9 (1): 123. DOI: https://doi.org/10.1186/1471-2180-9-123

Martinez JL (2009). Environmental pollution by antibiotics and by antibiotic resistance determinants. Environmental Pollution, 157 (11): 2893-2902. DOI: https://doi.org/10.1016/j.envpol.2009.05.051

Mazmanian SK, Liu CH, Tzianabos AO and Kasper DL (2005). An immunomodulatory molecule of symbiotic bacteria directs maturation of the host immune system. Cell, 122 (1): 107-118. DOI: https://doi.org/10.1016/j.cell.2005.05.007

Morotomi M, Nagai F and Watanabe Y (2012). Description of christensenella minuta gen. Nov., sp. Nov., isolated from human faeces, which forms a distinct branch in the order clostridiales, and proposal of christensenellaceae fam. Nov. International Journal of
Systematic \& Evolutionary Microbiology, 62 (1): 144-149. DOI: https://doi.org/10.1016/j.envpol.2009.05.051

Pan D and Yu Z (2014). Intestinal microbiome of poultry and its interaction with host and diet. Gut Microbes, 5 (1): 108-119. DOI: https://doi.org/ 10.4161/gmic.26945

Polansky O, Sekelova Z, Faldynova M, Sebkova A, Sisak F and Rychlik I (2015). Important metabolic pathways and biological processes expressed by chicken cecal microbiota. Applied \& Environmental Microbiology, 82 (5): 1569 . Available at:. DOI: https://doi.org/10.1128/AEM.03473-15

Qu A, Brulc JM, Wilson MK, Law BF, Theoret JR, Joens LA, Konkel ME, Angly F, Dinsdale EA and Edwards RA (2008). Comparative metagenomics reveals host specific metavirulomes and horizontal gene transfer elements in the chicken cecum microbiome, PLoS ONE. 3 (8): e2945. DOI: https://doi.org/10.1128/AEM.03473-15

Sears CL (2005). A dynamic partnership: Celebrating our gut flora. Anaerobe, $11 \quad$ (5): $247-251 . \quad$ DOI: https://doi.org/10.1016/j.anaerobe.2005.05.001

Shang Y, Kumar S, Oakley B and Kim WK (2018a). Chicken gut microbiota: Importance and detection technology. Frontiers in Veterinary Science, 5 (254). Available at: https://www.frontiersin.org/article/10.3389/fvets.2018.00254.

Shang Y, Kumar S, Oakley BandKim WK (2018b). Chicken gut microbiota: Importance and detection technology, 5 (254). Link Retrieved from journal Available at: https://www.frontiersin.org/article/10.3389/fvets.2018.00254.

Shin SM, Kwon JH, Lee SW, Kong HS, Lee SJ, Lee CK, Cho KH, Ha NJ and Kim KJ (2010). Immunostimulatory effects of cordyceps militaris on macrophages through the enhanced production of cytokines via the activation of NF-kappaB. Immune Netw, 10 (2): 55-63. DOI: https://doi.org/10.4110/in.2010.10.2.55

Spence C, Wells WG and Smith CJ (2006). Characterization of the primary starch utilization operon in the obligate anaerobe bacteroides fragilis: Regulation by carbon source and oxygen. Journal of Bacteriology, 188 (13): 4663. DOI: https://doi.org/10.1128/JB.00125-06

Stanley D, Geier MS, Denman SE, Haring VR, Crowley TM, Hughesm RJ and Moore RJ (2013). Identification of chicken intestinal microbiota correlated with the efficiency of energy extraction from feed. Veterinary Microbiology, 164 (1-2): 85-92. DOI: https://doi.org/10.1016/j.vetmic.2013.01.030

Videnska, Petra, Rahman, Md M, Faldynova, Marcela, Babak, Vladimir, Elsheimer M, Marta et al. (2014). Characterization of egg laying hen and broiler fecal microbiota in poultry farms in croatia, czech republic, hungary and slovenia. PLoS ONE, 9 (10): e110076. DOI: https://doi.org/10.1371/journal.pone.0110076

Wang CL, Chiang CJ, Chao YP, Yu B and Lee TT (2015). Effect of cordyceps militaris waster medium on production performance, egg traits and egg yolk cholesterol of laying hens. The Journal of Poultry Science. DOI: https://doi.org/10.2141/jpsa.0140191

Wang J, Liu YM, Cao W, Yao KW, Liu ZQ and Guo JY (2012). Antiinflammation and antioxidant effect of cordymin, a peptide purified from the medicinal mushroom cordyceps sinensis, in middle cerebral artery occlusion-induced focal cerebral ischemia in rats. Metabolic Brain Disease, 27 (2): 159-165. DOI: https://doi.org/10.1007/s11011-012-9282-1

Zhang C, Zhang M, Wang S, Han R, Cao Y, Hua W, Mao Y, Zhang X, Pang $X$ and Wei $C$ (2010). Interactions between gut microbiota, host genetics and diet relevant to development of metabolic syndromes in mice. Multidisciplinary Journal of Microbial Ecology, 4 (2): 232241. DOI: https://doi.org/10.1038/ismej.2009.112

Zhao L, Gang W, Siegel P, He C, Wang H, Zhao W, Zhai Z, Tian F, Zhao $J$ and Hao Z (2013). Quantitative genetic background of the host influences gut microbiomes in chickens. Scientific Reports, 3 (5): 1163. DOI: https://doi.org/10.1038/srep01163 